\title{
Exosomes Derived from Human Umbilical Cord Mesenchymal Stem Cells Promote Osteogenesis Through the AKT Signaling Pathway in Postmenopausal Osteoporosis
}

\section{Shi-wei Ren}

The Provincial Hospital Affiliated to Shandong First Medical University https://orcid.org/0000-00034457-0729

\section{Yang Song}

Shandong University Affiliated Hospital: Shandong Provincial Hospital

\section{Qing-run Zhu}

The Provincial Hospital Affiliated to Shandong First Medical University

\section{Min-gang He}

Shandong Tumor Hospital and Institute,Shandong First Medical University and Shandong Academy of Medical Sciences

\section{Jie Qiu}

The Provincial Hospital Affiliated to Shandong First Medical University

\section{Qiang Wang}

The Provincial Hospital Affiliated to Shandong First Medical University

\section{Feng Wang ( $\sim$ sdslyywangfeng@163.com )}

The Provincial Hospital Affiliated to Shandong First Medical University https://orcid.org/0000-00024565-8215

\section{Research article}

Keywords: postmenopausal osteoporosis, exosomes, human umbilical cord mesenchymal stem cells, osteogenesis

Posted Date: October 5th, 2021

DOl: https://doi.org/10.21203/rs.3.rs-903221/v1

License: (c) (1) This work is licensed under a Creative Commons Attribution 4.0 International License. Read Full License 


\section{Abstract}

\section{Background}

Postmenopausal osteoporosis (PMO) is a relatively common disease characterized by low bone mass and microstructural changes of trabecular bone. The reduced bone strength is caused a variety of complications, including fragility fracture and sarcopenia.

\section{Methods}

We used CCK-8 and EdU assays to evaluate cell proliferation rates. The osteogenesis effect was detected using ALP staining, alizarin red staining, and q-PCR. In vivo, the effects of exosomes derived from HUCMSCs were evaluated using HE staining, IHC staining and Masson staining. In addition, we explored the mechanism of exosomes and found that the AKT signaling pathway played an important role in osteogenesis and cell proliferation.

\section{Results}

This paper mainly explored the function of exosomes derived from human umbilical cord mesenchymal stem cells (HUC-MSCs) and provided a new strategy for the treatment of postmenopausal osteoporosis.

\section{Conclusions}

In conclusion, exogenous administration of exosomes can contribute to the treatment postmenopausal osteoporosis to a certain extent.

\section{Introduction}

With the development of society and technology, human life is increasingly extending in length, and most women's ages at menopause are approximately 45 to 55 years [1]. For a long time after menopause, the risks of various diseases increase. Postmenopausal osteoporosis (PMO) is a common disease in middleaged and old women, mainly due to the lack of estrogen, which decreases the content of bone and promotes bone structural changes[2]. This can lead to an increased risk of fractures, which can lead to pain, complications, decreased quality of life and even death, causing burdens on families and society[3]. However, the underlying mechanism has not been fully explored. As previously reported[4, 5], osteoblasts and osteoclasts, which participate in bone formation and bone resorption, respectively, are important for bone homeostasis during bone remodeling. In addition, most of the existing treatments such as bisphosphate are very expensive and have many side effects.

Recently, studies reported that exosomes derived from stem cells show great potential in tissue regeneration[6, 7]. Exosomes are extracellular vessels with diameters of approximately $40-100 \mathrm{~nm}$. Exosomes are released from a number of tissues and cells, mediating intercellular communication and transferring bioactive molecules, including DNA, microRNA, protein and lipids[8]. There were three 
communication modes between osteoblasts and osteoclasts: direct contact, paracrine pathway and growth factor deposition in bone matrix. Exosomes from mineralized osteoblasts as well as osteoclasts and osteoblasts can participate in bone remodeling by promoting osteogenesis or osteoclast formation[9]. As previously reported, miR-214 contained in osteoclast-derived exosomes promoted osteoclast production by macrophages in bone marrow stromal cells via the PI3K/Akt pathway; thus, miR-214 contained in osteoclast exosomes caused pathological destructive bone disease[9]. Exosomes derived from osteoclast precursors activate osteoblast differentiation, while exosomes derived from osteoclasts inhibit osteoblast formation. Therefore, exosomes play a major role in interventions for various diseases. Exosomes provide a potential, attractive and novel treatment for postmenopausal osteoporosis.

The phosphatidylinositol 3-kinase (PI3K) protein family is involved in the regulation of cell proliferation, differentiation, apoptosis and glucose transport[10,11]. Activated AKT regulates cell functions by phosphorylating downstream factors such as enzymes, kinases and transcription factors. In this paper, we explored the function and mechanism of exosomes derived from human umbilical cord mesenchymal stem cells (HUC-MSCs) and found that the AKT signaling pathway played an important role in osteogenesis and cell proliferation. This paper explored the function of exosomes derived from HUCMSCs and provided a new strategy for the treatment of postmenopausal osteoporosis.

\section{Material And Methods}

\subsection{Animal model and experiments}

All animal procedures and experiments were provided by the Animal Laboratory of the Provincial Hospital Affiliated to Shandong First Medical University. Animal experiments were conducted in accordance with the International Guidelines for Animal Research provided by the Council of International Medical Organizations (CIOMS) and approved by the Animal Ethics and Welfare Committee of the Provincial Hospital Affiliated to Shandong First Medical University. Female C57BL/6 mice $(20 \pm 2 \mathrm{~g})$ were housed under 12-h light/dark conditions, and food and water were offered ad libitum. The PMO model was established as previously reported. The mice were placed in the prone position, chloral hydrate was used for anesthesia, hair was removed, skin was disinfected, a midline dorsal skin incision was made, the ovaries were removed with scissors, and the enterocoelia were sutured in turn until the skin. The sham group underwent a midline incision, removal the adipose tissue around the ovaries, and closure of the incision. Antibiotics were given to prevent and treat infection after surgery, and the incisions were disinfected every day. Mice with PMO were randomly divided into four treatment groups: (1) PMO + PBS group; (2) PMO + exosome group; (3) PMO + bpV(phen) group; and (4) PMO + Exo + MK-2206 group. All mice were sacrificed at 8 weeks. Bones of the lower extremities were collected for subsequent experiments.

\subsection{Cell isolation and culture}


Cancellous bone was taken from the tibia and femur of experimental mice, periosteum and other soft tissues were stripped, and washed with sterile PBS 3 times. In the DMEM environment, cancellous bone was chopped to $0.5-1 \mathrm{~mm}^{3}$ size and then washed with PBS. Then, $0.25 \%$ trypsin was added and digested in a $37{ }^{\circ} \mathrm{C}$ constant temperature box for 20 minutes. Fetal bovine serum was terminated by digestion, and 5 times the volume of the red blood cell lysates was added. The lysates were centrifuged at $500 \mathrm{~g}$ for $5 \mathrm{~min}$, and the supernatants were removed. We added $1 \%$ collagenase type $1,37^{\circ} \mathrm{C}$ water bath to digest $1 \mathrm{~h}, 1000 \mathrm{r} / \mathrm{min} 5 \mathrm{~min}$ of centrifugation of the supernatants, and pellets were re-suspended in DMEM. We used 200 stainless steel mesh to filter and count cells. We made cell suspensions of $5 \times 10^{4} / \mathrm{ml}$, injected into sterile petri dishes, cultured in $5 \% \mathrm{CO}_{2}$ and $95 \% \mathrm{O}_{2}$ in the incubator at $37{ }^{\circ} \mathrm{C}$, and changed the culture medium every $48 \mathrm{~h}$. When cells were close to confluence, $0.25 \%$ trypsin was used to pass the cells.

\subsection{Extraction and identification of exosomes derived from HUC-MSCs}

HUC-MSCs were cultured for exosome extraction as previously reported. Exo-free FBS was used for HUCMSC culture. The culture supernatant of HUC-MSCs was collected and centrifuged at $300 \cdot g$ at $4^{\circ} \mathrm{C}$ for 10 $\mathrm{min}$. Then, the absorbed precipitate was centrifuged at $2000 \cdot \mathrm{g}$ for $30 \mathrm{~min}$, centrifuged at $10000 \cdot \mathrm{g}$ for $30 \mathrm{~min}$, and centrifuged at $140000 \cdot \mathrm{g}$ for $90 \mathrm{~min}$. The supernatants were removed, washed and precipitated with PBS, centrifuged again for $90 \mathrm{~min}$ at $140000 \mathrm{~g}$, the supernatants were removed, resuspended in $100 \mu \mathrm{L}$ PBS, and frozen at $-80^{\circ} \mathrm{C}$ until use. The morphology of exosomes derived from HUC-MSCs was observed using transmission electron microscopy (TEM). The size distribution was analyzed using dynamic light scattering (DLS). Specific exosome biomarkers, including Alixs, CD9 and CD81, were measured using western blotting assay.

\subsection{Exosome labeling with PKH-26}

PKH-26 red membrane dye was used for exosome labeling. Forty microliters of PKH-26 and $500 \mu \mathrm{L}$ of dilution buffer were mixed for exosome labeling. The prepared exosomes were cocultured with the mixture in the dark for $30 \mathrm{~min}$. Then, $10 \%$ FBS was used to stop the staining reaction. The labeled exosomes were extracted at $100,000 \cdot g$ for $1 \mathrm{~h}$ at $4{ }^{\circ} \mathrm{C}$ with ultracentrifugation. The supernatant was removed, and the labeled exosomes were resuspended in $100 \mu \mathrm{L}$ PBS and cocultured with BMSCs for 24 h.

\subsection{5-Ethynyl-2'-deoxyuridine (EdU) staining assay}

DNA replication activity was evaluated by an EdU Apollo567 in vitro assay kit (Solarbio, Beijing, China) according to the manufacturer's instructions to further determine the proliferation rate of cells. The logarithmic phase cells were cultured to the growth stage. According to the experimental specifications, the cells were cultured in medium at a 1000:1 ratio of dilution EdU solution for 4 hours.

Paraformaldehyde was used to fix cells for $20 \mathrm{~min}$. Then, $0.1 \%$ Triton X-100 was used for permeation for 5 min. After staining with DAPI, a confocal fluorescence microscope was used for imaging. 


\subsection{CCK-8 assay}

Cell proliferation was detected using the CCK-8 assay. According to the manufacturer's instructions, BMSCs were seeded in a 96-well plate at a density of 1000 cells per well. The cell plate was cultured in the incubator, and $10 \mu \mathrm{L}$ of CCK-8 solution was added into each well and incubated in the incubator for 4 hours at $0 \mathrm{~h}, 24 \mathrm{~h}, 48 \mathrm{~h}$ and $72 \mathrm{~h}$. An enzyme standard instrument was used to measure the absorbance at $450 \mathrm{~nm}$.

\subsection{Western blotting assay}

The bone tissues or cells were digested in RIPA lysate containing protease/phosphatase inhibitor and PMSF and centrifuged at $13800 \times g$ for 10 minutes at $4^{\circ} \mathrm{C}$. The supernatants were removed, the protein concentrations were measured using a BCA kit, loading buffer was added, and equal amounts of protein were electrophoresed using SDS-PAGE. The samples were concentrated at $120 \mathrm{~V}$ for $30 \mathrm{~min}$ and separated at $80 \mathrm{~V}$ for $1 \mathrm{~h}$. The proteins were transferred to PVDF membranes at a constant current of 200 $\mathrm{mA}$. The membranes were blocked with skimmed milk for $3 \mathrm{~h}$, and the primary antibody (p-AKT Thr308, $\mathrm{p}$ AKT Ser473, GAPDH) was used for incubation overnight at $4^{\circ} \mathrm{C}$. After rinsing with TBST, the secondary antibody was incubated at room temperature for $1 \mathrm{~h}$. After washing three times, the chemiluminescent signal was developed using ECL kit reagents.

\subsection{Alizarin red staining assay}

BMSCs with different interventions were cultured in six-well plates containing osteogenic differentiation medium (Cyagen, Suzhou, China). The osteogenic differentiation medium was changed every 3 days for 27 days. After washing the cells with PBS, the cells were fixed in 4\% paraformaldehyde for $30 \mathrm{~min}$. After the fixation solution was aspirated, the cell samples were washed with PBS twice, and alizarin red dye was added for 5 min of staining. After staining, the cells were washed with PBS twice. Finally, images were observed under a confocal microscope, and ImageJ was used to analyze the results.

\subsection{ALP staining assay}

BMSCs with different interventions were cultured in six-well plates containing osteogenic differentiation medium (Cyagen, Suzhou, China). The osteogenic differentiation medium was changed every 3 days for a week. After washing the cells with PBS, the cells were fixed in $4 \%$ paraformaldehyde for $30 \mathrm{~min}$. ALP dye was added for 15 min of staining. The cells were washed with PBS and observed under a confocal microscope. ImageJ was used to analyze the results.

\subsection{RNA extraction, reverse transcription and qPCR}

According to the manufacturer's instructions, total RNA was extracted from cancellous bone or cells using TRIzol reagent. The total RNA of each sample was reverse transcribed to cDNA using the ReverTra Ace Qpcr RT kit. The SYBR Green PCR master mix was used to conduct qRT-PCR in the Bio-Rad IQ5 real-time PCR system. The results were standardized with respect to U6 or $\beta$-actin, and gene expression analysis 
was performed using a $2^{-\triangle} \Delta \mathrm{Ct}$ approach. The primers for $\beta$-actin were designed and synthesized by BioSune Biological Co., Ltd.

\subsection{Statistical analysis}

SPSS software was used to analyze the data. All values are expressed as the mean \pm standard deviation. Differences of multiple groups were compared by one-way analysis of variance (Fisher's least significant difference [LSD]). A p value $<0.05$ was considered statistically significant.

\section{Results}

\subsection{Identification of exosomes derived from HUC-MSCs}

Exosomes derived from HUC-MSCs were extracted by ultracentrifugation and were identified. Transmission electron microscopy (TEM) observations of exosomes derived from HUC-MSCs revealed the presence of spherical vesicles that were either circular or typically cup-shaped (Fig. 1A). The size of exosomes derived from HUC-MSCs was analyzed using DSL, and the overall size distribution was 70 to $130 \mathrm{~nm}$, which was consistent with previous reports[8] (Fig. 1B). In addition, we confirmed the presence of markers, including TSG101 and CD9, from exosomes derived from HUC-MSCs using western blotting (Fig. 1C). Moreover, PKH26 fluorescently labeled exosomes were recorded in BMSCs. PKH26-labeled exosomes were observed in the cytoplasm (Fig. 1D).

\subsection{Effect of exosomes derived from HUC-MSCs on BMSC proliferation and osteogenesis}

Normal BMSCs and BMSCs extracted from PMO mice were cocultured with exosomes derived from HUCMSCs to explore the effect of exosomes. A CCK-8 assay was used to measure the proliferation rate of cells. The results showed that the proliferation rate of BMSCs extracted from PMO mice was reduced compared with that of the control group on Days 1,2 and 3. Exosomes derived from HUC-MSCs promoted the proliferation rate of BMSCs extracted from PMO mice (Fig. 2A and 2B). In addition, we explored the osteogenesis effect of exosomes, and an alizarin red staining assay was performed. The results showed that the osteogenesis of BMSCs extracted from PMO mice was reduced compared with that of the control group. Exosomes derived from HUC-MSCs significantly improved the osteogenesis of BMSCs extracted from PMO mice (Fig. 2C and 2D). Additionally, osteogenic genes, including BMP2 and RunX2, were analyzed by qPCR. The results showed that the expression of BMP2 and RunX2 was inhibited in BMSCs extracted from the PMO mouse group, and exosomes reversed the effect of PMO to promote the expression of BMP2 and RunX2 (Fig. 2E and 2F).

\subsection{Mechanism of exosomes derived from HUC-MSCs in PMOs}


As we previously reported, the AKT signaling pathway showed important significance in the regulation of bone metabolism[12]. Therefore, we explored the expression of key genes in the AKT signaling pathway using qPCR. The results showed that the expression of PI3KCA and PI3KR1 was inhibited in BMSCs extracted from the PMO mouse group, and exosomes promoted the expression of these genes (Fig. 3A and 3B). PTEN is an important gene in the AKT signaling pathway[11]. We also investigated the expression of PTEN in vitro. PTEN was upregulated in the PMO group, and exosomes inhibited the expression of PTEN (Fig. 3C). In addition, we explored the expression of AKT; two phosphorylation sites, Thr 308 and Ser 473, were detected. The results showed that p-AKT was downregulated in BMSCs extracted from the PMO mouse group, and exosomes promoted the phosphorylation of AKT (Fig. 3D, 3E and $3 F$ ).

\subsection{Exosomes derived from HUC-MSCs promoted BMSC proliferation and osteogenesis through the AKT signaling pathway}

We next verified the effect and mechanism of exosomes derived from HUC-MSCs. MK-2206, an inhibitor to inhibit the expression of AKT[12], and bpV(Phen)[10], a PTEN inhibitor, were used to investigate the mechanism of exosomes on cell proliferation and osteogenesis. The cell proliferation rate was detected by EdU staining. The results showed that the positive EdU cells were promoted when cocultured with bpV(Phen) in BMSCs extracted from PMO mice, and the positive EdU cells were inhibited when cocultured with MK-2206 in BMSCs extracted from PMO mice (Fig. 4A and 4B). Osteogenesis was evaluated using ALP and alizarin red staining. The results showed that no significant difference was found between the control and PMO + bpV(phen) groups in ALP and alizarin red staining (Fig. 4C-4F). When treated with MK2206, the osteogenesis effect was significantly inhibited in the PMO + Exo group (Fig. 4C-4F). The positive EdU cells, alizarin red and ALP area were evaluated using ImageJ software.

\subsection{Exosomes derived from HUC-MSCs promoted osteogenesis in PMO mice through the AKT signaling pathway}

We verified the effect of exosomes derived from HUC-MSCs using a PMO mouse model. The results of HE staining showed that the bone tissue in the PMO group was significantly reduced compared with that of the control group. The exosomes derived from HUC-MSCs increased the bone volume, and the bone volume was significantly increased in the $\mathrm{PMO}+\mathrm{bpV}$ (phen) group compared with the PMO group. MK2206 significantly inhibited the effect caused by exosomes to reduce bone volume (Fig. 5A and 5B). In addition, we performed Masson staining to evaluate the new bone formation rate. The results showed that new bone formation was significantly inhibited in the PMO group, and exosomes rescued this effect to promote new bone formation. Additionally, bpV(phen) inhibited PTEN to activate the AKT signaling pathway to promote new bone formation, and MK-2206 inhibited AKT expression to inhibit bone formation (Fig. 5A and 5C). Moreover, we explored IHC staining to detect the expression of p-AKT. The results showed that the expression of p-AKT was inhibited in the PMO group, and exosomes promoted 
the expression of p-AKT when compared with the PMO group. In addition, bpV(phen) promoted the expression of p-AKT, and MK-2206 significantly inhibited the expression of p-AKT (Fig. 5A and 5D).

\section{Discussion}

Postmenopausal osteoporosis is a problem faced by middle-aged and elderly women around the world and is characterized by bone fragility and skeletal deterioration $[3,13,14]$. A high risk of fragility fractures was the main complication in PMO patients[15], and the medical costs of fracture treatment has brought heavy economic burdens to society. Therefore, how to prevent and solve this problem has been studied by many academic researchers. Existing effective drug treatments, including denosumab and teriparatide, are costly[2]. Therefore, it is necessary to find new treatments that are cost-effective for PMO patients. In this experiment, exosomes derived from HUC-MSCs were used for PMO. Additionally, we investigated the mechanism of exosomes derived from HUC-MSCs to promote osteogenesis in vitro and in vivo through the AKT signaling pathway.

As previously reported, exosomes derived from mesenchymal stem cells showed potential in tissue regeneration[16, 17]. Zhang et al.[18] reported that exosomes derived from MSCs can promote osteochondral regeneration and that the effect of MSC-exosomes coordinated multiple cell types through regulation of the AKT signaling pathway and ERK signaling by AMPCP. Additionally, we previously reported that exosomes derived from MSCs can reduce apoptosis of osteocytes in glucocorticoid-induced osteonecrosis of the femoral head via the miR-21-PTEN-AKT signaling pathway [19]. All these findings indicated the potential of exosomes in skeletal regeneration. In this study, we extracted exosomes from the human umbilical cord, which is considered useless in obstetric operations. However, in our opinion, the advantages of umbilical cord mesenchymal stem cells were obvious, including easy access to sources, substantial presence of stem cells, and others. Therefore, we used HUC-MSCs for exosome extraction.

Additionally, we explored the effect of exosomes derived from HUC-MSCs. We mainly focused on the cell proliferation and osteogenesis effects. As we previously reported, exosomes derived from HUC-MSCs showed anti-apoptosis effects in glucocorticoid-induced osteonecrosis of the femoral head in rats[19]. Yang et al.[20] demonstrated that exosomes derived from human umbilical cord mesenchymal stem cells inhibited apoptosis through miR-1263 delivery to regulate the Hippo signaling pathway to prevent apoptosis in disuse osteoporosis. In this study, we explored the effect of exosomes derived from HUCMSCs on cell proliferation and osteogenesis effects. We found that exosomes derived from HUC-MSCs can rescue the cell proliferation and osteogenesis of BMSCs extracted from PMO mice.

The mechanisms of exosomes have been widely explored in previous studies[21-23]. Exosomal miRNAs accounted for more than $40 \%$ of exosomes[24]. Therefore, previous studies reported that exosomal miRNAs play important roles in cellular functions, including apoptosis, cell migration and proliferation based on epigenetic modification[25-27]. In our study, we explored the mechanisms of exosomes at the protein level. As previously reported[11], PI3KCA promoted the phosphorylation of PI3K, thereby 
promoting the expression of osteogenic genes, while PTEN dephosphorylated PI3K and reduced its osteogenic effect. We found that exosomes derived from HUC-MSCs activated the AKT signaling pathway to regulate cell proliferation and osteogenesis.

\section{Conclusions}

Additionally, we used a PTEN inhibitor and AKT inhibitor to lose and gain, respectively, the function of AKT signaling to explore the effect of exosomes. HE, Masson and IHC staining of the mouse model also verified the effect of AKT on osteogenesis.

\section{Abbreviations}

PMO Postmenopausal osteoporosis

HUC-MSCs Human umbilical cord mesenchymal stem cells

DMEM Dulbecco's modified eagle medium

BMSCs Bone mesenchymal stem cells

PI3K Phosphatidylinositol 3-kinase

AKT Protein kinase $B$

HE Hematoxylin-eosin

PTEN Phosphatase and tensin homolog deleted on chromosome ten

IHC Immunohistochemistry

\section{Declarations}

\section{Ethics approval and consent to participate}

Animal experiments were conducted in accordance with the International Guidelines for Animal Research provided by the Council of International Medical Organizations (CIOMS) and approved by the Animal Ethics and Welfare Committee of the Provincial Hospital Affiliated to Shandong First Medical University.

\section{Consent for publication}

Shi-wei Ren/Yang Song/Qing-run Zhu/Min-gang He/Jie Qiu/ give my consent for information about myself to be published in Stem Cell Research by Professor Feng Wang .I understand that the information will be published without my name attached, but that full anonymity cannot be guaranteed. 
I understand that the text and any pictures or videos published in the article will be freely available on the internet and may be seen by the general public. The pictures, videos and text may also appear on other websites or in print, may be translated into other languages or used for commercial purposes.I have been offered the opportunity to read the manuscript. Signing this consent form does not remove my rights to privacy.

\section{Availability of data and materials}

All the data and materials are available when published online.

\section{Competing interests}

All the authors declared that no competing of interests.

\section{Funding}

None.

\section{Authors' contributions}

All animal experiments were performed by Shi-wei Ren. Feng Wang and Qiang Wang designed this study .Yang Song and Shi-wei Ren write the manuscript. The cell experiment was done by Qing-run Zhu. Min-gang He and Jie Qiu carried out the statistical analysis. All authors read and approved the final manuscript.

\section{Acknowledgements}

I like to thank my co-authors for their contributions to this study and the writing of this manuscript.

\section{References}

1. Compston JE, McClung MR, Leslie WD (2019) Osteoporosis. Lancet (London, England) 393:364-376

2. Rachner TD, Khosla S, Hofbauer LC (2011) Osteoporosis: now and the future. Lancet (London, England) 377:1276-1287

3. Black DM, Rosen CJ (2016) Clinical Practice. Postmenopausal Osteoporosis. The New England journal of medicine $374: 254-262$

4. Guler-Yuksel M, Hoes JN, Bultink IEM, Lems WF (2018) Glucocorticoids, Inflammation and Bone. Calcified tissue international

5. Lee NK, Sowa H, Hinoi E, Ferron M, Ahn JD, Confavreux C, Dacquin R, Mee PJ, McKee MD, Jung DY, Zhang Z, Kim JK, Mauvais-Jarvis F, Ducy P, Karsenty G (2007) Endocrine regulation of energy metabolism by the skeleton. Cell 130:456-469

6. Liu J, Li D, Wu X, Dang L, Lu A, Zhang G (2017) Bone-derived exosomes. Current opinion in pharmacology 34:64-69 
7. Marote A, Teixeira FG, Mendes-Pinheiro B, Salgado AJ (2016) MSCs-Derived Exosomes: Cell-Secreted Nanovesicles with Regenerative Potential. Frontiers in pharmacology 7:231

8. van Niel G, D'Angelo G, Raposo G (2018) Shedding light on the cell biology of extracellular vesicles. Nature reviews. Molecular cell biology

9. Li D, Liu J, Guo B, Liang C, Dang L, Lu C, He X, Cheung HY, Xu L, Lu C, He B, Liu B, Shaikh AB, Li F, Wang L, Yang Z, Au DW, Peng S, Zhang Z, Zhang BT, Pan X, Qian A, Shang P, Xiao L, Jiang B, Wong CK, Xu J, Bian Z, Liang Z, Guo DA, Zhu H, Tan W, Lu A, Zhang G (2016) Osteoclast-derived exosomal miR-214-3p inhibits osteoblastic bone formation. Nature communications 7:10872

10. Jeong SH, Kim HB, Kim MC, Lee JM, Lee JH, Kim JH, Kim JW, Park WY, Kim SY, Kim JB, Kim H, Kim JM, Choi HS, Lim DS (2018) Hippo-mediated suppression of IRS2/AKT signaling prevents hepatic steatosis and liver cancer. The Journal of clinical investigation 128:1010-1025

11. Chalhoub N, Baker SJ (2009) PTEN and the PI3-kinase pathway in cancer. Annual review of pathology 4:127-150

12. Kuang M-j, Zhang W-h, He W-w, Sun L, Ma J-x, Wang D, Ma X-I (2019) Naringin regulates bone metabolism in glucocorticoid-induced osteonecrosis of the femoral head via the Akt/Bad signal cascades. Chemico-biological interactions 304:97-105

13. Hinton PS, Nigh P, Thyfault J (2015) Effectiveness of resistance training or jumping-exercise to increase bone mineral density in men with low bone mass: A 12-month randomized, clinical trial. Bone 79:203-212

14. Li J, Chen X, Lu L, Yu X (2020) The relationship between bone marrow adipose tissue and bone metabolism in postmenopausal osteoporosis. Cytokine \& growth factor reviews

15. Amin S, Achenbach SJ, Atkinson EJ, Khosla S, Melton LJ, 3rd (2014) Trends in fracture incidence: a population-based study over 20 years. Journal of bone and mineral research : the official journal of the American Society for Bone and Mineral Research 29:581-589

16. Beretti F, Zavatti M, Casciaro F, Comitini G, Franchi F, Barbieri V, La Sala GB, Maraldi T (2018) Amniotic fluid stem cell exosomes: Therapeutic perspective.

17. Cabral J, Ryan AE, Griffin MD, Ritter T (2018) Extracellular vesicles as modulators of wound healing. Advanced drug delivery reviews

18. Zhang S, Chuah SJ, Lai RC, Hui JHP, Lim SK, Toh WS (2017) MSC exosomes mediate cartilage repair by enhancing proliferation, attenuating apoptosis and modulating immune reactivity. Biomaterials 156:16-27

19. Kuang MJ, Huang Y, Zhao XG, Zhang R, Ma JX, Wang DC, Ma XL (2019) Exosomes derived from Wharton's jelly of human umbilical cord mesenchymal stem cells reduce osteocyte apoptosis in glucocorticoid-induced osteonecrosis of the femoral head in rats via the miR-21-PTEN-AKT signalling pathway. Int J Biol Sci 15:1861-1871

20. Yang BC, Kuang MJ, Kang JY, Zhao J, Ma JX, Ma XL (2020) Human umbilical cord mesenchymal stem cell-derived exosomes act via the miR-1263/Mob1/Hippo signaling pathway to prevent apoptosis in disuse osteoporosis. Biochemical and biophysical research communications 
21. Hervera A, De Virgiliis F, Palmisano I, Zhou L, Tantardini E, Kong G, Hutson T, Danzi MC, Perry RB, Santos CXC, Kapustin AN, Fleck RA, Del Rio JA (2018) Reactive oxygen species regulate axonal regeneration through the release of exosomal NADPH oxidase 2 complexes into injured axons. 20:307-319

22. Su T, Xiao Y, Xiao Y, Guo Q, Li C, Huang Y, Deng Q, Wen J, Zhou F, Luo XH (2019) Bone Marrow Mesenchymal Stem Cells-Derived Exosomal MiR-29b-3p Regulates Aging-Associated Insulin Resistance. 13:2450-2462

23. Qu L, Ding J, Chen C, Wu ZJ, Liu B, Gao Y, Chen W, Liu F, Sun W, Li XF, Wang X, Wang Y, Xu ZY, Gao L, Yang Q, Xu B, Li YM, Fang ZY, Xu ZP, Bao Y, Wu DS, Miao X, Sun HY, Sun YH, Wang HY, Wang LH (2016) Exosome-Transmitted IncARSR Promotes Sunitinib Resistance in Renal Cancer by Acting as a Competing Endogenous RNA. Cancer cell 29:653-668

24. Zhang J, Li S, Li L, Li M, Guo C, Yao J, Mi S (2015) Exosome and Exosomal MicroRNA: Trafficking, Sorting, and Function. Genomics, Proteomics \& Bioinformatics 13:17-24

25. Zhou W, Fong MY, Min Y, Somlo G, Liu L, Palomares MR, Yu Y, Chow A, O'Connor STF, Chin AR, Yen Y, Wang Y, Marcusson EG, Chu P, Wu J, Wu X, Li AX, Li Z, Gao H, Ren X, Boldin MP, Lin PC, Wang SE (2014) Cancer-secreted miR-105 destroys vascular endothelial barriers to promote metastasis. Cancer cell 25:501-515

26. Fan Q, Yang L, Zhang X, Peng X, Wei S, Su D, Zhai Z, Hua X, Li H (2018) The emerging role of exosome-derived non-coding RNAs in cancer biology. Cancer letters 414:107-115

27. Xiao J, Pan Y, Li XH, Yang XY, Feng YL, Tan HH, Jiang L, Feng J, Yu XY (2016) Cardiac progenitor cellderived exosomes prevent cardiomyocytes apoptosis through exosomal miR-21 by targeting PDCD4. Cell death \& disease 7:e2277

\section{Figures}


A

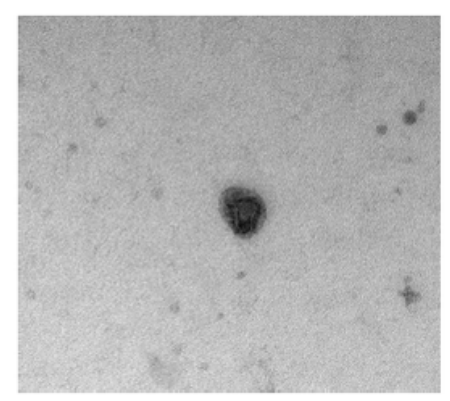

B

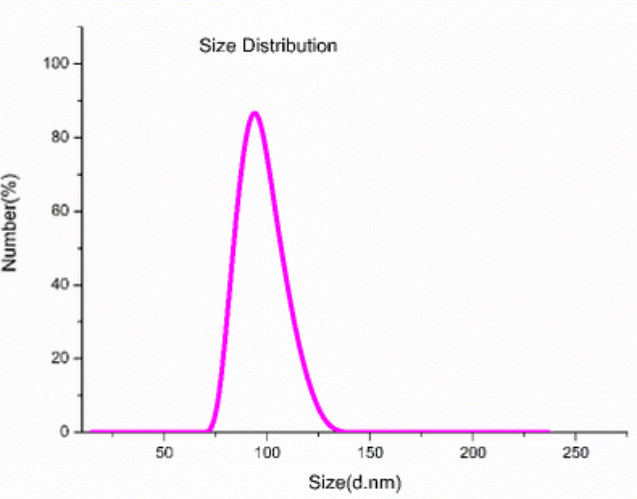

C
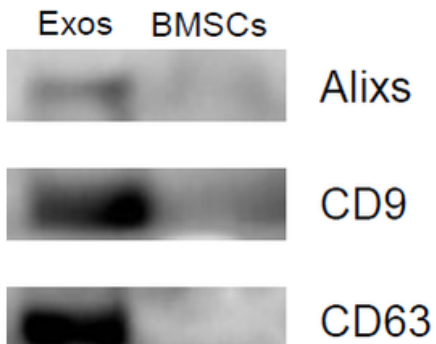

D

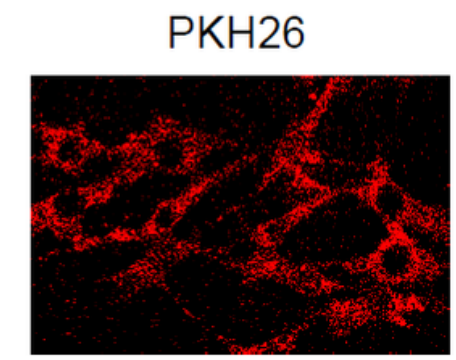

DAPI

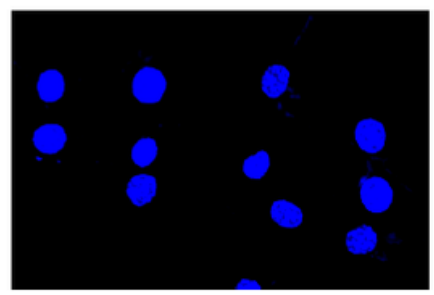

Merge

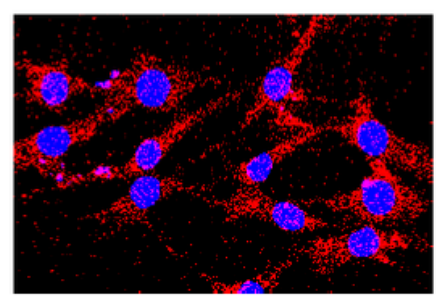

Figure 1

(A) The size and morphology of exosomes derived from HUC-MSCs were determined using transmission electron microscopy (TEM). (B) The size distribution of exosomes derived from HUC-MSCs was scattered and identified using DLS. (C) The surface biomarkers Alixs, CD9 and CD63 were detected using western blot to identify exosomes derived from HUC-MSCs. (D) The exosomes derived from HUC-MSCs were labeled with $\mathrm{PKH} 26$. 
A

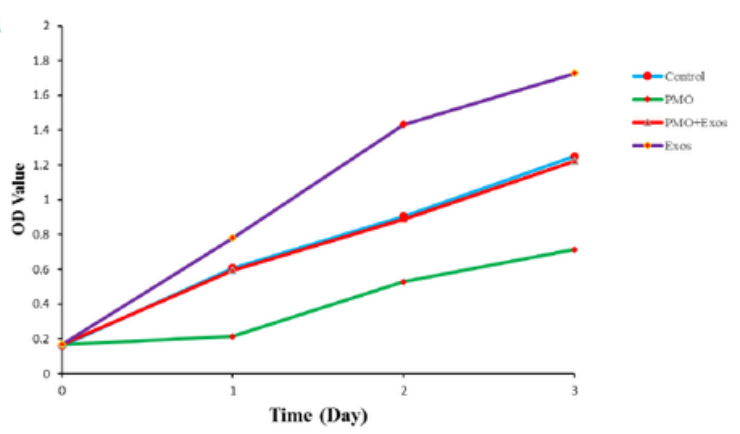

C

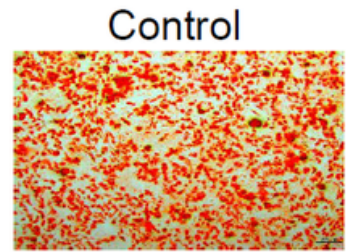

D

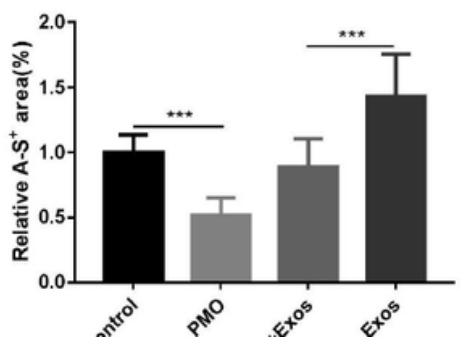

B

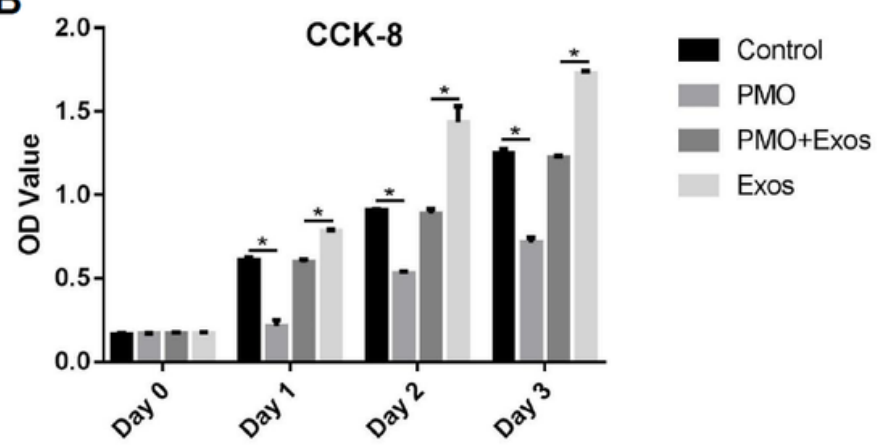

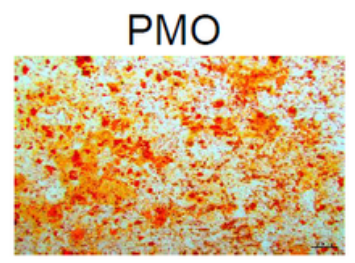

E

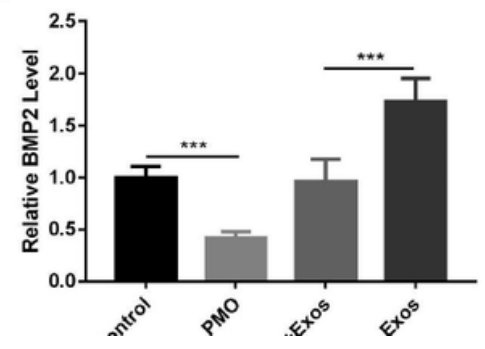

$\mathrm{PMO}+\mathrm{ExOS}$

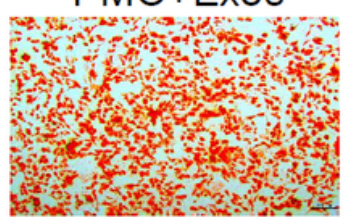

$\mathbf{F}$

\section{Figure 2}

(A) CCK-8 was used to detect the proliferation rates of BMSCs extracted from PMO mice. (B) The CCK-8 results were calculated using SPSS software, and significant differences are labeled. (C) Alizarin red assay was performed to evaluate the effect of osteogenesis. (D) The results of alizarin red staining were calculated using ImageJ software. (E) qPCR was performed to detect the expression of BMP2. (F) qPCR was performed to detect the expression of RunX2. 
A

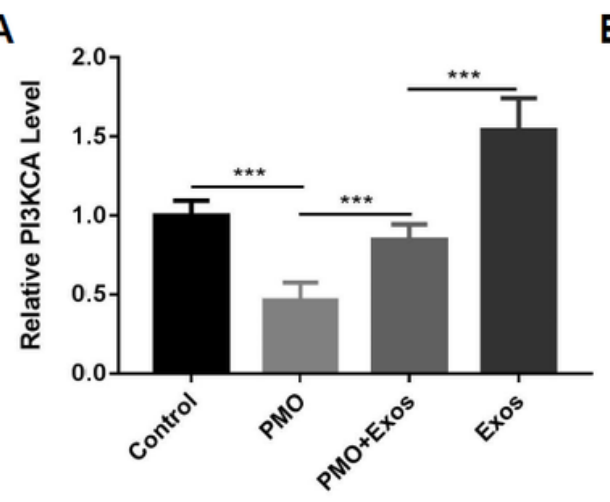

D

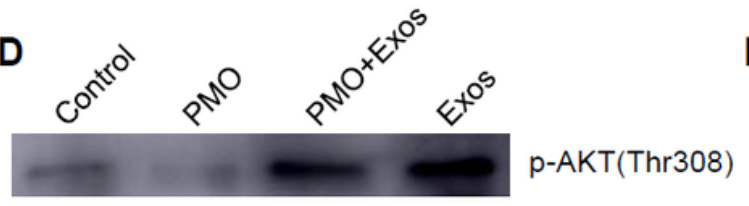

p-AKT(Ser473)

(2) GAPDH
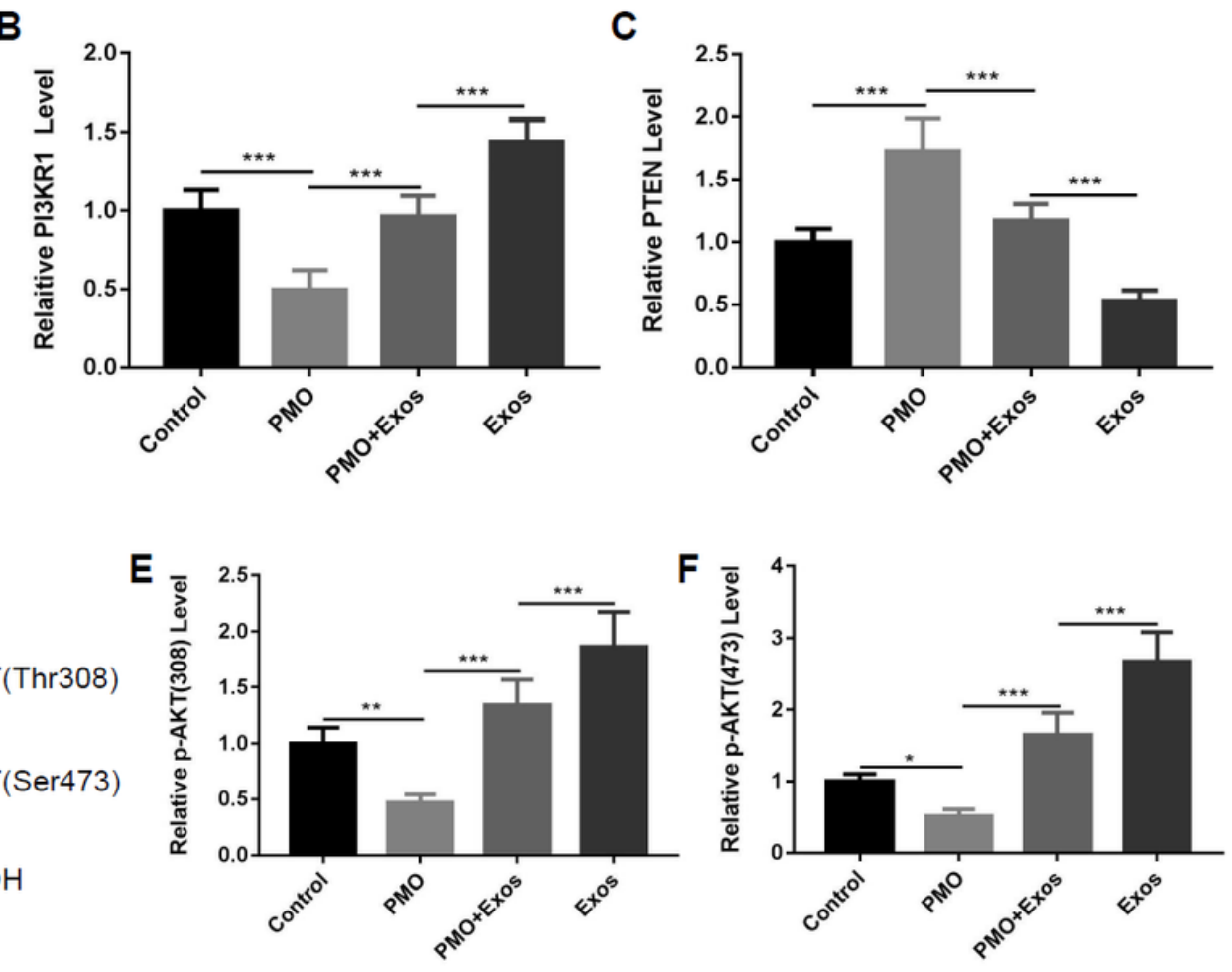

Figure 3

The expression of PI3KCA (A), PI3KR1 (B) and PTEN (C) was measured using qPCR in the control group, PMO group, PMO+exosome group and exosome group. (D) The expression of p-AKT (Thr308) and p-AKT (Ser 473) was detected using western blotting in the control group, PMO group, PMO+exosome group and exosome group. The results of p-AKT (Thr308) (E) and p-AKT (Ser 473) (F) were calculated using Image J software. 


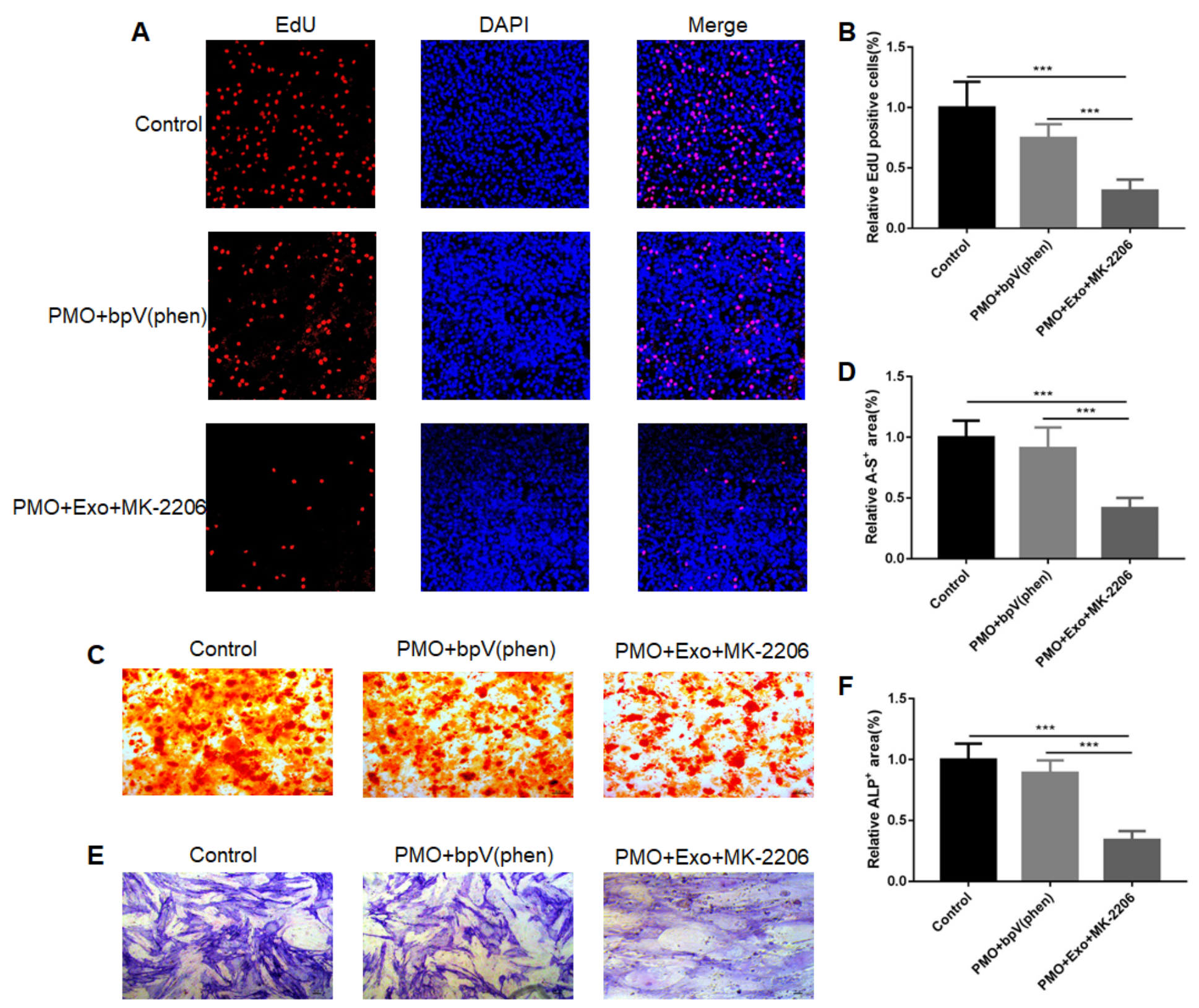

Figure 4

(A) Cell proliferation was evaluated using EdU staining between the control group, $\mathrm{PMO}+\mathrm{bpV}$ (phen) group and PMO+Exo+MK-2206. (B) The EdU-positive cells were calculated using ImageJ. (C) Alizarin red assay was performed to evaluate osteogenesis between different groups. (D) Quantitative calculation of alizarin red-positive areas. (E) We performed an ALP assay to evaluate osteogenesis between different groups.

(F) Quantitative calculation of ALP-positive areas. 
A
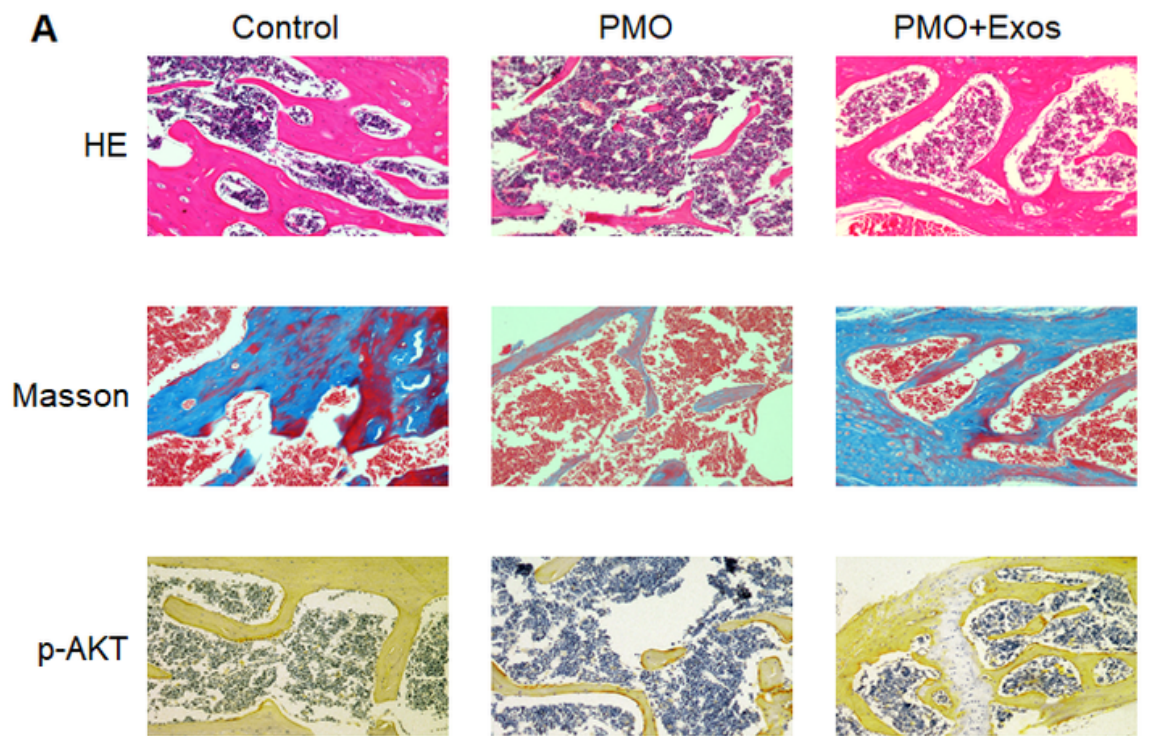

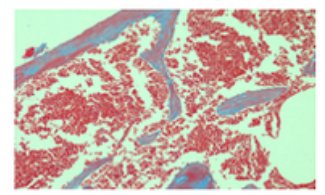

PMO
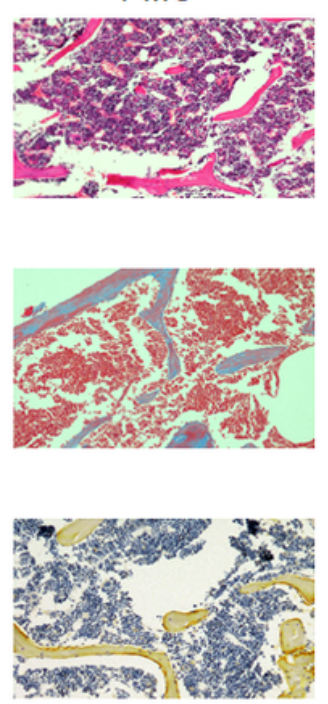

PMO+Exos

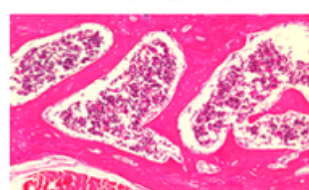

लमx.?

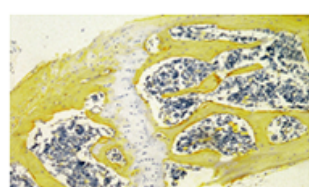

PMO+bpV(phen)

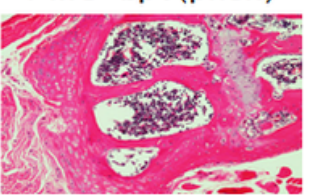

PMO+ExO+MK-2206
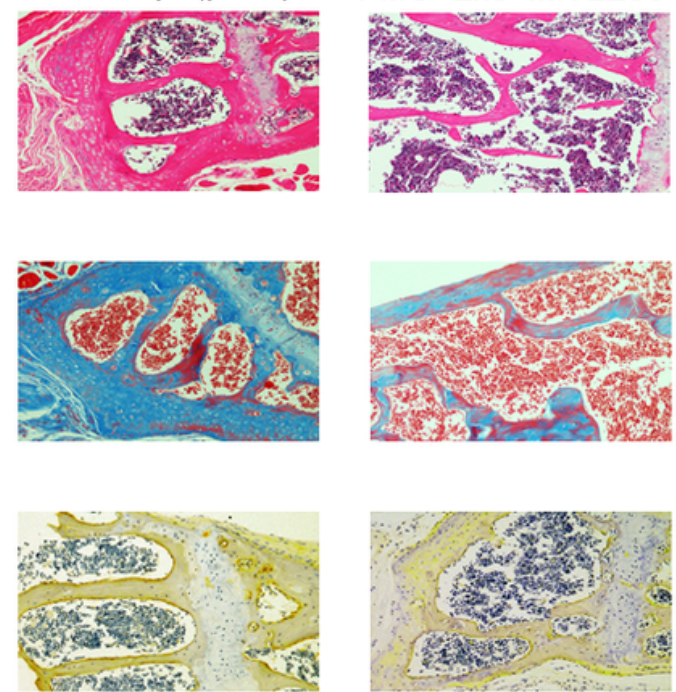

B

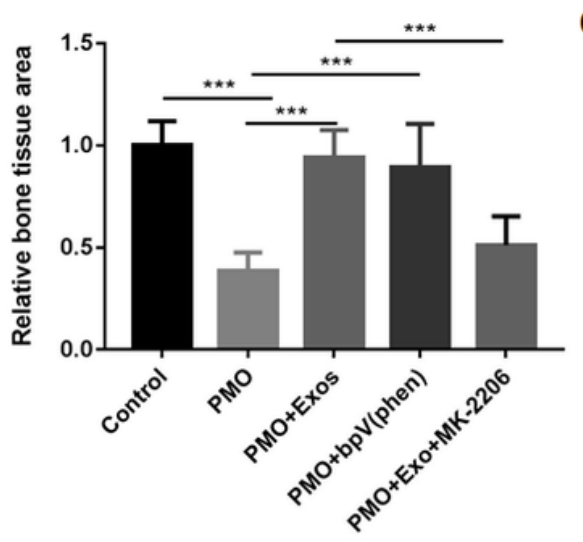

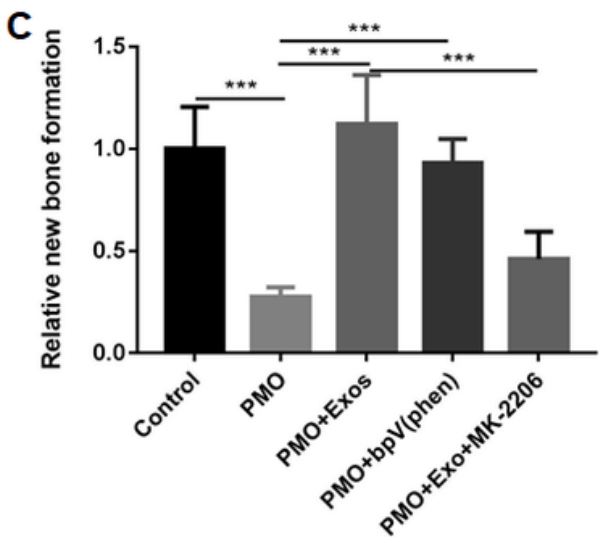

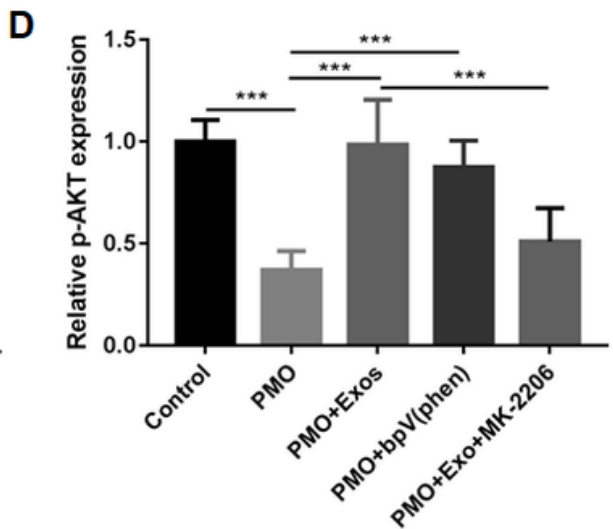

\section{Figure 5}

(A) HE staining, Masson staining and IHC (p-AKT) staining were performed to investigate osteogenesis between different groups. (B) The bone volume was calculated by HE staining using ImageJ. (C) The new bone formation rate was calculated by HE staining using ImageJ. (D) The expression of p-AKT was calculated by HE staining using ImageJ. 